\title{
Original Article (short paper) \\ Effects of the platelet-rich fibrin associated with physical exercise in a model of median nerve compression.
}

\author{
Gladson Ricardo Flor Bertolini ${ }^{1}$, Camila Mayumi Martin Kakihata ${ }^{1}$, Ana Luiza Peretti ${ }^{1}$, Giovanni Ribeiro Bernar- \\ dino $^{1}$, Jhenifer Karvat ${ }^{2}$, José Luis da Conceição Silva ${ }^{1}$, Rose Meire Costa Brancalhão ${ }^{1}$, Lucinéia de Fátima Chasko \\ Ribeiro $^{1}$ \\ ${ }^{1}$ Universidade Estadual do Oeste do Paraná, Unioeste, Cascavel, PR, Brazil; ${ }^{2}$ Univer- \\ sidade Federal de Santa Catarina, UFSC, Florianópolis, SC, Brazil.
}

\begin{abstract}
Aims: Platelet-Rich Fibrin (PRF) is a new and promising technique for tissue repair, however, there is still a gap about its action on peripheral nerve injury, as well as its association with physical exercise. Thus, the aim of this study was to evaluate the effects of the PRF associated with physical exercise in a model of median nerve compression. Methods: 42 rats, divided into: Control, non-injured limb, and other six groups with nerve lesion (Control lesion; Treated with PRF; Treadmill walking; Free swimming; PRF and treadmill; PRF and swimming). The lesion model was performed with median nerve compression. To obtain the PRF, $1.5 \mathrm{~mL}$ of blood was centrifuged and the fibrin clot positioned directly over the compression region. The exercise protocols were performed during 2 weeks. The evaluations performed were grip strength tests and histologic analysis. Results: both, grip strength and histomorphometric evaluations (fiber numbers and axon density) did not present significant differences between the treated and lesion groups, however, in the morphological evaluation it was possible to distinguish characteristics of the repair process for the treated groups. Conclusion: a slight qualitative improvement was observed for the treated groups, especially when it was associated PRF with free swimming.
\end{abstract}

Keywords: muscle strength; median nerve; exercise therapy; fibrin.

\section{Introduction}

Compression of the median nerve can occur at several points of its path, generating different denominations; as in the pronator syndrome, where there is compression when it passes between the two heads of the pronator teres muscle or under the proximal border of the flexor arch of the fingers. Usually shows pain in the volar portion of the forearm, with paresthesias radiating to the thumb, $2^{\text {nd }}$ and $3^{\text {rd }}$ fingers and radial half of the $4^{\text {th }}$ finger. In anterior interosseous syndrome, which is a motor branch, patients commonly present with weakness or absence of thumb flexor longus function, with pain in the forearm along the nerve ${ }^{1}$. However, the most common compressive neuropathy of the upper limb is carpal tunnel syndrome. Such a condition is responsible for substantial costs to society in terms of lost productivity and treatment values ${ }^{2}$. It is a prevalent condition that affects millions of individuals, causing chronic pain, altered sensitivity and tenar atrophy ${ }^{3}$.

Conditions such as pregnancy, hypothyroidism, hemodialysis, diabetes and inflammatory processes, may generate increased pressure within the carpal tunnel, consequently the compression of the median nerve. High incidence has also been observed in patients with occupations requiring repetitive movements, especially those with prolonged flexion or extension of the wrist, strong grip, torque maneuvers, or exposure to vibration ${ }^{4-6}$, including athletes as enduro motorcyclists ${ }^{7}$.

In those cases the first option is the conservative treatment, are suggested as a forms: splint, local and oral steroid, therapeutic ultrasound ${ }^{2}$, low-level laser therapy and carpal bone mobilizations; exercises and ergonomic changes, although there are controversies ${ }^{8-10}$; nor about the use of physical activity, as a means of reducing symptoms in upper limbs of typists ${ }^{9}$. Although indications are that exercise-induced analgesia results from both increased pain threshold and increased levels of endogenous opioids in the blood ${ }^{11}$. When conservative treatment is not efficient, usually the surgical procedure for the release of flexor retinaculum is used, however, individual characteristics should be taken into account for a suitable rehabilitation process ${ }^{12}$.

By other side platelet concentrates are used in surgeries or injury sites to stimulate, ameliorate and accelerate tissue repair in most medical fields, particularly in sports medicine and orthopedic surgery ${ }^{13}$, with indications for use in central nervous lesions ${ }^{14}$ and peripheral, despite controversies regarding their results ${ }^{15-17}$. A new generation of platelet concentrates is Platelet-Rich Fibrin (PRF), which has a simple, quick and inexpensive preparation to produce large amounts of fibrin clots and membranes in a short time. The technique does not require anticoagulant or bovine thrombin, only a tabletop centrifuge and a quick handling, due to blood clotting ${ }^{13}$. During PRF processing, platelets are activated and degranulate massively, which implies significant release of cytokines ${ }^{18}$, pro (IL-1 $\beta$, IL-6 e TNF- $\alpha$ ) and anti-inflammatory (IL-4) ${ }^{19}$, worth highlighting several other factors that may interfere with the nervous repair process (PDGF-AB, TGF- $\beta 1$, VEGF, EGF) ${ }^{20}$.

Since experimental protocols in animals aid in the scientific advancement, by the homogeneity of lesions, as well as in the therapeutic ones, and still given the contradictory results of platelet concentrates, as well as the absence of studies associating their 
use with exercise, such as the low-intensity resistance that is a commonly used form in different levels of health care, like swimming $^{21}$ and running on a treadmill ${ }^{22}$. This study aims to evaluate if the PRF with the exercise promotes increased strength and neural recovery after experimental median nerve compression.

\section{Methods}

Thirty-six male Wistar rats, mean weight of $363.4 \pm 59.6 \mathrm{~g}$, and age $12 \pm 2$ weeks, were used in a $12 \mathrm{~h}$ photoperiod, $24 \pm 1^{\circ} \mathrm{C}$, with water and feed ad libitum, kept in polypropylene boxes with dimensions of $410 \times 340 \times 160 \mathrm{~mm}$. The animals were separated into groups consisting of six animals randomly, into seven groups:

- $\mathrm{C}$-Absolute control, non-injured limb of the animals, being composed randomly by 1 animal from each group;

- CL - Control lesion, submitted to nerve compression and untreated;

- NCPRF - Nerve compression and PRF;

- NCTW - Nerve compression and treadmill walking;

- NCFS - Nerve compression and free swimming;

- NCPRF+TW - Nerve compression, PRF and treadmill walking;

- NCPRF+FS - Nerve compression, PRF and free swimming.

The research was conducted according to the international standards of ethics in animal experimentation, and approved for accomplishment by the Committee of Ethics in Animal Use of the Universidade Estadual do Oeste do Paraná (Unioeste), under protocol 05612.

\section{Median Nerve Compression Protocol}

In order to perform the median nerve compression, the median nerve was exposed above the elbow joint, after exposure, nerve compression was performed by a Catgut 4.0 chrome in 4-point, it was used the mocking model, with a distance of approximately $1 \mathrm{~mm}$, in the region proximal to the right elbow, according with the model of Chen et al. ${ }^{21}$. The animals were anesthetized with ketamine hydrochloride solution $(50 \mathrm{mg} / \mathrm{kg})$ and xylazine hydrochloride $(10 \mathrm{mg} / \mathrm{kg})$ to perform The nerve compression surgical procedure. nerve compression was always performed by the same examiner.

\section{Protocol for obtaining PRF}

For the preparation of the PRF, $1.5 \mathrm{~mL}$ of blood was withdrawn via cardiac puncture from each animal ${ }^{15}$. Immediately after collection, the sample was placed into eppendorf sterile tubes (without anticoagulant) for centrifugation, at $3000 \mathrm{rpm}$, with a force of approximately $400 \mathrm{G}$, for 10 minutes. The PRF was removed from the middle layer of the centrifuged sample, between the red part (below) and the plasma (above). Then the fibrin clot was positioned directly over the median nerve, in the compression region. Both the PRF collection and the placement on the nerve compression site were performed by the same examiner.

\section{Treadmill Protocol}

One of the forms used, as treatment of the animals was the treadmill (Insight $\AA$, Ribeirão Preto - Brazil), at a speed of 10 $\mathrm{m} / \mathrm{min}$, for 10 minutes/day for NCTW and NCPRF+TW ${ }^{22}$. And, similarly to swimming, it was also performed in a period of five days of training prior to the nerve compression procedure, and then again from the $3^{\text {rd }}$ to the $14^{\text {th }} \mathrm{PO}$. The animals were placed for 10 minutes on the treadmill at standard speed, aiming to adapt them to the treadmill, in the five days before the lesion protocol.

\section{Swimming Protocol}

Another form of treatment was low intensity swimming, without overload, that is, the animals were free to perform swimming. In order to perform the swimming protocol, the animals were placed in a $200 \mathrm{~L}$ water tank with a depth of $60 \mathrm{~cm}$ and a water temperature between $30-32^{\circ} \mathrm{C}$. The animals, of NCFS and NCPRF+FS, started the swimming exercise five days before the surgery (as a form of training), interrupting shortly after the surgical procedure, restarting from the $3^{\text {rd }}$ to the $14^{\text {th }}$ day after the surgical procedure, totaling 12 days of therapy. Both during training, when during the treatment period, the swimming time was 10 minutes/day ${ }^{(23)}$.

\section{Functional Evaluation by the Grip Test}

For the evaluation of muscle strength, a rats grip strength gauge was used (Insight ${ }^{\circledR}$ - Ribeirão Preto, Brazil), consisting of a triangular arm coupled to a force transducer. To perform the assessment, the animals were gently secured by the tail and allowed to grasp on a grid attached to a force transducer. The animal was drawn by the tail, with increasing firmness, until it lost the grip. At this moment the maximum force exerted, recorded on the equipment was evaluated. Use of the left limb was prevented, temporarily, by wrapping with adhesive tape. In each evaluation the test was repeated three times and the mean value was used. The evaluations occurred prior to the injury (EV1) and the beginning of the treatment $\left(3^{\text {rd }} \mathrm{PO}-\mathrm{EV} 2\right)$, in the $7^{\text {th }}(\mathrm{EV} 3)$ and $15^{\text {th }}$ (one day after the end of the forms of therapy - EV4) PO.

\section{Histomorphometric evaluation}

Before decapitation, with the anesthetized animal, it was dissected and removed $1 \mathrm{~cm}$ from the median nerve distal to the compression procedure, fixed in 4\% paraformaldehyde in PBS ( $\mathrm{pH} 7.4$ ) for 48 hours (hrs) and then stored in alcohol $70 \%$ until histological processing. The fragments were post-fixed in $2 \%$ osmium tetroxide in PBS, dehydrated, diaphanized, infiltrated and embedded in paraffin to obtain cross-sections of the nerve with a thickness of $5 \mu \mathrm{m}$, and stained with hematoxylin and eosin. 
The histological slides were assembled, photomicrographically with a 100x objective (4 fields), and analyzed with Image Pro-plus 6.0 program for the following variables: counted the number of axons per field $\left(14700 \mu \mathrm{m}^{2}\right)$ and then analyzed the neuron density (number of neurons per square millimeter). For analysis of the morphometric data a blind evaluator was used, in relation to the groups.

\section{Data analysis}

The results were expressed and analyzed using descriptive and inferential statistics. The Shapiro-Wilk test was used and later ANOVA mixed model was used for comparison of the functional evaluation and unidirectional ANOVA for morphometric comparison, in both the Tukey post-test was used, in all cases the level of significance was $5 \%$.

\section{Results}

\section{Grip Assessment}

Regarding the grip strength, there were significant differences $(\mathrm{F}(1,9 ; 65,6)=62,0 ; \mathrm{p}<0,001)$. Being all injury groups, smaller than control $(p<0,01)$, but not different from each other $(p>0.05)$, with EV1 being higher than EV2-EV4 $(\mathrm{p}<0.001)$ (Table 1).

\section{Histomorphometric evaluation}

In the comparison of fiber numbers by visual field, a significant difference was observed between groups $(\mathrm{F}(6,28)=102,4$, $\mathrm{p}<0.0001)$, being that $\mathrm{C}$ presented higher values than the other groups. Result that was repeated when the axons density was evaluated $(\mathrm{F}(6,28)=102,4, \mathrm{p}<0.0001)($ table 2$)$.

Table 1. Presentation of the numerical data, on average and standard deviation, of the gripping force (g). The groups (absolute control (C), control lesion (CL), Nerve compression and PRF (NCPRF), Nerve compression and treadmill walking (NCTW), Nerve compression and free swimming (NCFS), Nerve compression, PRF and treadmill walking (NCPRF+TW) and Nerve compression, PRF and free swimming (NCPRF+FS)) are presented in columns and the evaluations (evaluation 1 (EV1), evaluation 2 (EV2), evaluation 3 (EV3) and evaluation 4 (EV4)) in the different lines.

\begin{tabular}{cccccccc}
\hline & $\mathbf{C}$ & $\mathbf{C L}$ & NCPRF $^{*}$ & NCTW* $^{*}$ & NCFS* & NCPRF+TW* & NCPRF+FS* \\
\hline EV1 & $138,6 \pm 27,8$ & $149,3 \pm 46,8$ & $135,2 \pm 43,1$ & $161,7 \pm 58,9$ & $158,5 \pm 36,5$ & $148,5 \pm 46,1$ & $173,0 \pm 69,5$ \\
& $148,1 \pm 42,5$ & $0 \pm 0$ & $0 \pm 0$ & $0 \pm 0$ & $13,5 \pm 33,0$ & $44,3 \pm 76,4$ & $9,3 \pm 15,4$ \\
\multirow{2}{*}{ EV3 $^{\#}$} & $183,6 \pm 75,9$ & $0 \pm 0$ & $0 \pm 0$ & $0 \pm 0$ & $37,3 \pm 91,4$ & $56,3 \pm 93,3$ & $72,8 \pm 113,6$ \\
& $174,9 \pm 43,6$ & $0 \pm 0$ & $0 \pm 0$ & $0 \pm 0$ & $9,4 \pm 23,1$ & $49,7 \pm 77,1$ & $50,6 \pm 76,4$ \\
\hline
\end{tabular}

* Significant difference when comparing with group C. \# significant difference when comparing with $\mathrm{EV} 1 . \mathrm{F}(1,9 ; 65,6)=62,0 ; \mathrm{p}<0,001$.

Table 2. Histomorphometric evaluation of median nerves, for the different groups, in the variables: total number of morphologically intact axons $(\mathrm{N})$ and axonal density (D) considering the area of $0.0896 \mathrm{~mm}^{2}$. The groups (absolute control (C), control lesion (CL), Nerve compression and PRF (NCPRF), Nerve compression and treadmill walking (NCTW), Nerve compression and free swimming (NCFS), Nerve compression, PRF and treadmill walking (NCPRF+TW) and Nerve compression, PRF and free swimming (NCPRF+FS) are presented in columns.

\begin{tabular}{cccccccc}
\hline & $\mathbf{C}$ & $\mathbf{C L}$ & NCPRF & NCTW & NCFS & NCPRF+TW & NCPRF+FS \\
\hline $\mathrm{N}$ & $511.8 \pm 100.0$ & $13.2 \pm 3.8^{*}$ & $15 \pm 3.5^{*}$ & $15.2 \pm 3.0^{*}$ & $25.2 \pm 26.9^{*}$ & $24.4 \pm 26.8^{*}$ & $26.4 \pm 18.8^{*}$ \\
$\mathrm{D}$ & $5712.0 \pm 1115.9$ & $147.3 \pm 42.8^{*}$ & $167.4 \pm 38.7^{*}$ & $169.7 \pm 33.8^{*}$ & $281.3 \pm 299.8^{*}$ & $272.3 \pm 298.7^{*}$ & $294.7 \pm 209.6^{*}$ \\
\hline
\end{tabular}

* Significant difference when comparing with group C. $F(6,28)=102,4, p<0.0001$.

\section{Morphological analysis}

The control group (Figures $1 \mathrm{~A}$ and $\mathrm{B}$ ) showed a characteristic morphology of peripheral nervous tissue, with intact nerve fibers with marked myelin and axon sheath, presence of Schwann cells and connective envelopes (endoneurium, perineurium and epineurium) intact. While the lesion group (Figure 1C) was different, with signs of alteration in the nerve fiber, identified by the presence of a large amount of empty spaces delimited by the endoneurium and many cell nuclei, possibly Schwann cells and inflammatory cells. In addition to hypercellularity, there was an increase in the number of blood vessels (not shown). In the lesion + fibrin group (figure 1D), it maintained the morphological characteristics of the nerve found in the lesion group.
The two groups that only performed physical exercise, the treadmill group (figure 1E) and swimming (figure 1F), also showed signs of alterations in the nervous fiber, with apparent hypercellularity, however, with fewer empty spaces when compared with the injury group; In addition, these groups had few fibers in regeneration in the periphery, being these, specifically in the treadmill group, with smaller diameter when compared to the control.

When associating the exercises with fibrin, the treadmill + fibrin group (figure $1 \mathrm{G}$ ), although it presented some regenerated fibers, these were in smaller quantity with greater presence of cellular nuclei. While an improvement in tissue appearance was evident in the swimming + fibrin group (figure $1 \mathrm{H}$ ), because despite the signs of hypercellularity and nerve damage, the group presented a higher number of regenerated fibers in the periphery of the nerve. 
Figure 1: Photomicrographs of median nerves of Wistar rats, cross section, Hematoxylin and Eosin staining. In A, Control group and B in greater increase, with intact peripheral nerve fibers (arrow) and cellular nuclei (NC). C, Injury group, with presence of empty spaces delimited by endoneurium (leaked arrow) and hypercellularity (NC). In D, Injury + Fibrin group, showing the same characteristics of empty spaces (arrow cast) and increase of cellular nuclei ( $\mathrm{NC})$ and some fibers in regeneration in the periphery $(*)$. E, treadmill group, with presence of voids (arrow cast), also evidenced in the group Swimming (F). In G, treadmill + Fibrin group also denoting the previous aspects, with high number of cellular nuclei (NC). H, group Swimming + Fibrin, but with its peripheral fibers having larger diameter and quantity of regenerated fibers $(*)$.
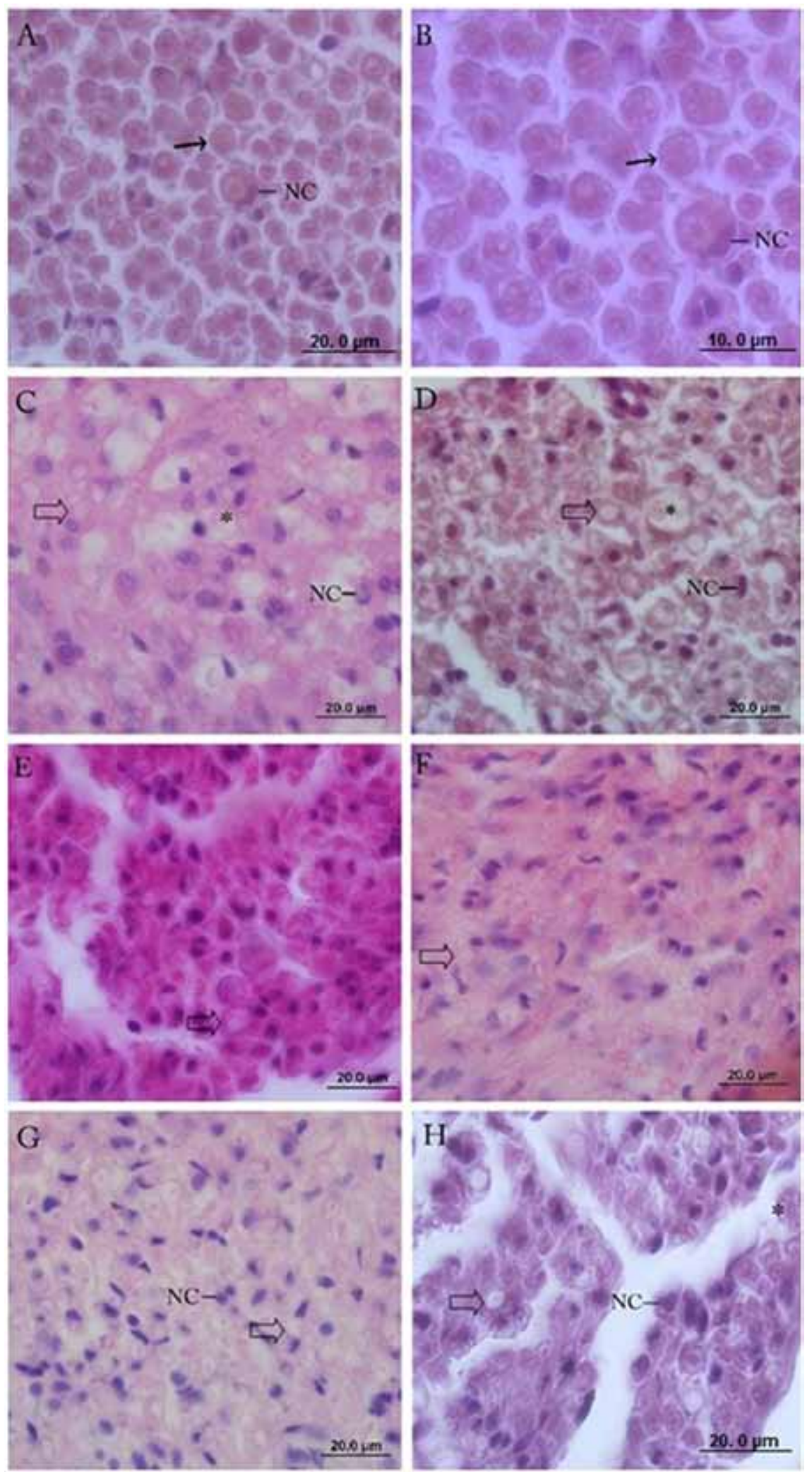


\section{Discussion}

Platelets are sources of cytokines and growth factors, with important functions in the tissue repair process. Thus, some platelet concentrates have been used in order to reduce pain, functional recovery and antibacterial activity. However, since the available information is still preliminary with respect to doses, half-life and interference from other resources, more research is generally required to establish therapeutic efficacy ${ }^{24}$. Similarly the physical exercise also presents controversies and there is ignorance with respect to the intensity and correlations with factors important for the tissue repair ${ }^{25}$. Thus the present study aimed to evaluate the association of the PRF with physical exercise in animals submitted to median nerve compression.

Axonal regeneration does not always allow adequate functional recovery, resulting in changes in normal motor control and fine sensitivity ${ }^{26}$, thus, studies that seek alternatives to improve quality and accelerate rehabilitation are important. For the production of median nerve compression, the model presented by Chen et al. ${ }^{21}$, which was based on the Bennett and Xie model ${ }^{27}$, performing a catgut 4.0 chromed wire at 4 points, with an approximate distance of $1 \mathrm{~mm}$, in the median nerve, in the proximal region to the right elbow. The aforementioned authors observed that the model generates, in the animal, painful symptoms and decreased motor function, but without producing self-mutilation, only limb protection, which begins around the $3^{\text {rd }}$ day. Thus, the beginning of the exercise protocols respected the $3^{\text {rd }} \mathrm{PO}$, as a milestone for the beginning of rehabilitation treatment.

For the PRF preparation, $1.5 \mathrm{ml}$ of blood were removed from each animal, as according to Ehrenfest, Lemo, Jimbo, Sammartino $^{28}$, not more than $10 \%$ of the total animal blood volume should be exceeded, requiring about 2 weeks for replacement, since for rats of approximately $250 \mathrm{~g}$ there is about $16 \mathrm{ml}$, ie $1.5 \mathrm{ml}$ is a safe volume. For the PRF production the use of anticoagulants and blood activators is not required, only requiring centrifugation to separate the components of the blood and to discard the elements considered useless (mainly the red cells) and to obtain and concentrate the elements that can be useful for applications (fibrinogen/fibrin, platelets, growth factors, leukocytes and other forms of circulating cells in plasma) $)^{13,29}$.

After blood withdrawal the sample was placed into sterile tubes to centrifuge at $3000 \mathrm{rpm}$ with approximately $400 \mathrm{G}$, for 10 minutes. This fact may have influenced in the results, since according to Eren, Gürkan, Atmaca, Dönmez, Atilla ${ }^{30}$ centrifuge for 12 minutes has higher VEGF levels than only 10 minutes. The PRF was removed from the middle layer of the centrifuged sample, between the red part (below) and the plasma (above), which is in agreement with the findings of Nishimoto et al. ${ }^{31}$ about higher concentrations of mononuclear cells, platelets and growth factors in the yellow-red interface area. However, the isolated effect of PRF was not observed in the functional or morphological evaluation. According to Fioravanti, Frustaci, Armellin, Condò, Arcuri, Cerroni ${ }^{32}$ the products derived from plasma, are safe and deliver several growth factors for soft tissue repair, but there is a wide range of clinical results.

Lichtenfels, Colomé, Sebben, Braga-Silva ${ }^{33}$ who also using Wistar rats performed a $5 \mathrm{~mm}$ excision of the sciatic nerve and used as a bridge a $12 \mathrm{~mm}$ silicone conduit filled with FPR, report that there was no functional improvement (sciatic functional index) without evolution in histomorphometric parameters. Senses et al. ${ }^{34}$ used FPR in a section and suture model, or removal of a $5 \mathrm{~mm}$ fragment, observed that there was less morphometric and functional recovery with the use of PRF. Although there are studies with the use of platelet-rich fibrin in the treatment of peripheral nerve injury, such as those cited above, but there is a gap with regard to its association with physical exercise such as swimming and running on treadmills experienced in the present study.

Physical exercise, although there are controversies about its use in peripheral nerve injuries, presents itself as an instrument widely used in the rehabilitation process ${ }^{35}$. The study by ArbatPlana, Torres-Espín, Navarro, Udina ${ }^{36}$ showed that running on a treadmill reduced the destruction of perineuronal nets after experimental injury in ischiatic rats; the changes were proportional to the intensity of exercise protocol, with the lightest form occurring at a speed of $10 \mathrm{~cm} / \mathrm{s}$, in two daily series of 30 minutes, with a 10 minute interval, for 5 days; that is, a protocol that was much more aggressive than that used in the present study, in which it was used about $16.7 \mathrm{~cm} / \mathrm{s}$ for 10 minutes. Asensio-Pinilla, Udina, Jaramillo, Navarro ${ }^{37}$ observed that the association of treadmill walking, $5 \mathrm{~m} / \mathrm{min}$ in two 30 -minute series, with electrostimulation had cumulative effect and the isolated modalities in the initial recovery were superior. Using a 4-series protocol of $20 \mathrm{~m} / \mathrm{min}$ for 2 minutes, intervals for 5 minutes, along 2 weeks, Brandt et al. ${ }^{38}$ observed that exercise, even performed after about 3 weeks of injury/repair, showed a greater recovery of the motoneurons and reinnervation of the muscle fibers compared to control, however they report doubts about the sensory reinnervation.

Cobianchi, Casals-Diaz, Jaramillo, Navarro ${ }^{39}$ performed an electrostimulation, then the sciatic nerve section of rats, and from the $3^{\text {rd }}$ day after the injury, started running on a treadmill, with increasing intensities from $10 \mathrm{~cm} / \mathrm{s}$, for 1 hour, to 5 days. They observed that the electrical stimulation produced an increase in the levels of BDNF, NGF, GDNF and NT-3 mRNA, whereas on treadmill walking there was a reduction in the levels of neurotrophins, but, associating the early electrostimulation, there was reduction of the pain with acceleration in the sensory and motor recovery. Similarly, in the present study, immediate intraoperative therapy (PRF) and two forms of postoperative treatment (treadmill and swimming) were used, but in a different way from that reported above. No functional change was seen in treated groups, only initial grip recovery was observed in 2 animals from each of the groups in which physical exercise was associated with PRF, but without statistical differences; however, for the mean percentage values compared to the baseline values within the same group, it was possible to observe that in the second evaluation, there was abolition of the values for the injury control and for the treatments with PRF and running on mat, for the swimming group the initial reduction was $91 \%$, $70 \%$ for PRF + TW, and 94\% for PRF + FS. These results may have occurred due to the training that occurred prior to the injury procedure in these groups. At the end of the experiment the reductions were $94 \%$ for the swimming group, $66 \%$ for the $\mathrm{PRF}+\mathrm{TW}$, and $70 \%$ for the PRF $+\mathrm{FS}$, indicating that even 
non-significant there were slight returns of the grip strength for these groups. Although not significant, there were different mean values between the absolute control and the others, especially for the group in which treatment with PRF and swimming was associated, which although not evaluated, may indicate a predominance of non-dominant limbs (since the absolute control group was used as the left limb of the other groups) or animal stress because it had already performed the evaluation in the contralateral limb.

There were also no significant differences in morphometric evaluations, however, for the number of intact cells, it can be observed that when comparing with the injury control, there were increases in mean percentages of $13 \%$ for PRF, $15 \%$ for treadmill running, $90 \%$ for swimming group, $85 \%$ for PRF association group and running, and still 100\% for the PRF association and swimming group. however, small qualitative morphological alterations, representative of tissue recovery, were observed for the treated groups. To the PRF group, there were characteristics of inflammatory process with macrophages and giant cells presence. In the groups in which physical exercise was performed, some fibers presented regeneration characteristics, with apparent hypercellularity, however, with fewer empty spaces when compared with the injury group. To the group PRF+SF there were more nerve fibers with well-defined sheath and axon.

Teodori et al. ${ }^{40}$ evaluated free swimming for 30 minutes daily, in two weeks, performed from the first day or after 14 days of injury, as a treatment for sciatic nerve compressive injury in rats, a lesion similar to the one performed in the present study. They observed that both protocols were efficient for both functional and histomorphometric recovery. In the present study, the swimming protocol was also free, but with less exercise time, and although it did not show significant differences in the functional and morphometric data, the histological findings also pointed to recovery, especially when swimming was preceded by treatment with PRF. It is highlighted as limitations that morphometric variables such as axon and nerve fiber diameters, however, due to tissue destruction, were not possible to be performed, in addition the absence of evaluation of neurotrophic factors that may have been stimulated with the forms of therapeutics used. Thus, it is suggested that future studies explore longer exercise times associated with the use of PRF and also perform analyzes of neurotrophic factors, because the association of PRF with exercises is still an unexplored subject with respect to recovery in cases of peripheral nerve injury.

\section{Conclusion}

The isolated or associated use of the PRF with physical exercises did not produce statistical quantitative functional or morphometric changes in the median nerve compression model, however, in the morphological evaluation it was possible to distinguish defined characteristics of the repair process for the treated groups, it means a slight qualitative improvement, and the association of PRF with free swimming presented more advanced tissue recovery, but PRF with treadmill running also presented indications for functional recovery of grip strength.

\section{References}

1. Dang AC, Rodner CM. Unusual compression neuropathies of the forearm, part II: median nerve. J Hand Surg Am. 2009;34(10):1915-20.

2. Bickel KD. Carpal tunnel syndrome. J Hand Surg. 2010;35(1):147-52.

3. Newington L, Harris EC, Walker-Bone K. Carpal tunnel syndrome and work. Best Pr Res Clin Rheumatol. 2015;29(3):440-53.

4. Lo SL, Raskin K, Lester H, Lester B. Carpal tunnel syndrome: a historical perspective. Hand Clin. 2002;18(2):211-7.

5. Palmer KT, Bovenzi M. Rheumatic effects of vibration at work. Best Pract Res Clin Rheumatol. 2015;39(3):424-39.

6. Tanik N, Sarp U, Ucar M, Celikbilek A, Balbaloglu O, Ak H, et al. Pain, depression and sleep disorders in patients with diabetic and nondiabetic carpal tunnel syndrome: a vicious cycle. Arq Neuropsiquiatr. 2016;74(3):207-11.

7. Sabeti-Aschraf M, Serek M, Pachtner T, Auner K, MacHinek M, Geisler M, et al. The Enduro motorcyclist's wrist and other overuse injuries in competitive Enduro motorcyclists: A prospective study. Scand J Med Sci Sport. 2008;18(5):582-90.

8. Aroori S, Spence RAJ. Carpal tunnel syndrome. Ulster Med J. 2008;77(1):6-17.

9. Bernaards CM, Ariëns GAM, Knol DL, Hildebrandt VH. The effectiveness of a work style intervention and a lifestyle physical activity intervention on the recovery from neck and upper limb symptoms in computer workers. Occup Env Med. 2011;68(4):265-72.

10. Malanotte JA, Kakihata CMM, Karvat J, Brancalhão RMC, Ribeiro L de FC, Bertolini GRF. Jumping in aquatic environment after sciatic nerve compression: nociceptive evaluation and morphological characteristics of the soleus muscle of Wistar rats. Einstein (São Paulo). 2017;15(1):77-84.

11. Koltyn KF. Analgesia following exercise. A review. Sport Med. 2000;29(2):85-98.

12. Yucel H. Factors affecting symptoms and functionality of patients with carpal tunnel syndrome: a retrospective study. J Phys Ther Sci. 2015;27(4):1097-101.

13. Ehrenfest DMD, Andia I, Zumstein MA, Zhang C-Q, Pinto NR, Bielecki T. Classification of platelet concentrates (Platelet-Rich Plasma- PRP, Platelet-Rich Fibrin-PRF) for topical and infiltrative use in orthopedic and sports medicine: current consensus, clinical implications and perspectives. Muscles Ligaments Tendons J. 2014;4(1):3-9.

14. Shen Y-X, Fan Z-H, Zhao J-G, Zhang P. The application of platelet-rich plasma may be a novel treatment for central nervous system diseases. Med Hypotheses. 2009;73(6):1038-40.

15. Ding X-G, Li S-W, Zheng X-M, Hu L-Q, Hu W-L, Luo Y. The effect of platelet-rich plasma on cavernous nerve regeneration in a rat model. Asian J Androl. 2009;11(2):215-21.

16. Elgazzar RF, Mutabagani MA, Abdelaal SE, Sadakah AA. Platelet rich plasma may enhance peripheral nerve regeneration after cyanoacrylate reanastomosis: a controlled blind study on rats. Int J Oral Maxillofac Surg. 2008;37(8):748-55.

17. Piskin A, Kaplan S, Aktas A, Ayyildiz M, Raimondo S, Aliç T, et al. Platelet gel does not improve peripheral nerve regeneration: 
an electrophysiological, stereological, and electron microscopic study. Microsurgery. 2009;29(2):144-53.

18. Dohan DM, Choukroun J, Diss A, Dohan SL, Dohan AJJ, Mouhyi J, et al. Platelet-rich fibrin (PRF): A second-generation platelet concentrate. Part II: Platelet-related biologic features. Oral Surgery, Oral Med Oral Pathol Oral Radiol Endodontology. 2006;101(3):e45-50.

19. Dohan DM, Choukroun J, Diss A, Dohan SL, Dohan AJJ, Mouhyi J, et al. Platelet-rich fibrin (PRF): A second-generation platelet concentrate. Part III: Leucocyte activation: A new feature for platelet concentrates? Oral Surgery, Oral Med Oral Pathol Oral Radiol Endodontology. 2006;101(3):e51-55.

20. Su CY, Kuo YP, Tseng YH, Su CH, Burnouf T. In vitro release of growth factors from platelet-rich fibrin (PRF): a proposal to optimize the clinical applications of PRF. Oral Surgery, Oral Med Oral Pathol Oral Radiol Endodontology. 2009;108(1):56-61.

21. Volpi FS, Casarolli LM, Pudell C, Menon T, Ciena AP, Alves ÉPB, et al. Effects of remobilization in two weeks of swimming on the soleus muscle of rats submitted to immobilization. Rev Bras Med do Esporte. 2008;14(3):168-70.

22. Natali LH, da Silva TS, Ciena AP, Padoin MJ, Alves EPB, Aragão FA, et al. Effects of running on treadmill in soleus muscles of rats shortened by immobilization. Rev Bras Med do Esporte. 2008;14(6):490-3.

23. Chen J, Lue J, Lin L, Huang C, Chiang RP, Chen C, et al. Effects of pre-emptive drug treatment on astrocyte activation in the cuneate nucleus following rat median nerve injury. Pain. 2010;148(1):158-66.

24. De Pascale MR, Sommese L, Casamassimi A, Napoli C. Platelet derivatives in regenerative medicine: an update. Transfus Med Rev. 2015;29(1):52-61.

25. English AW, Wilhelm JC, Ward PJ. Exercise, neurotrophins, and axon regeneration in the PNS. Physiology. 2014;29(6):437-45.

26. Allodi I, Udina E, Navarro X. Specificity of peripheral nerve regeneration: interactions at the axon level. Prog Neurobiol. 2012;98(1):16-37.

27. Bennett GJ, Xie YK. A peripheral mononeuropathy in rat that produces disorders of pain sensation like those seen in man. Pain. 1988;33(1):87-107.

28. Ehrenfest DMD, Lemo M, Jimbo R, Sammartino M. Selecting a relevant animal model for testing the in vivo effects of Choukroun's platelet-rich fibrin (PRF): Rabbit tricks and traps. Oral Surg Oral Med Oral Pathol Oral Radiol Endod. 2010;110(4):413-6.

29. Liao H-T, Marra KG, Rubin JP. Application of platelet-rich plasma and platelet-rich fibrin in fat grafting: basic science and literature review. Tissue Eng Part B Rev. 2014;20(4):267-76.

30. Eren G, Gürkan A, Atmaca H, Dönmez A, Atilla G. Effect of centrifugation time on growth factor and MMP release of an experimental platelet-rich fibrin-type product. Platelets. 2016;27(5):427-32.

31. Nishimoto S, Fujita K, Sotsuka Y, Kinoshita M, Fujiwara T, Kawai K, et al. Growth factor measurement and histological analysis in platelet rich fibrin: a pilot study. J Maxillofac Oral Surg. 2015;14(4):907-13.
32. Fioravanti C, Frustaci I, Armellin E, Condò R, Arcuri C, Cerroni L. Autologous blood preparations rich in platelets, fibrin and growth factors. Oral Implantol (Rome). 2015;8(4):96-113.

33. Lichtenfels M, Colomé L, Sebben AD, Braga-Silva J. Effect of platelet rich plasma and platelet rich fibrin on sciatic nerve regeneration in a rat model. Microsurgery. 2013;33(5):383-90.

34. Şenses F, Önder ME, Koçyiğit ID, Kul O, Aydin G, Inal E, et al. Effect of Platelet-Rich Fibrin on peripheral nerve regeneration. J Craniofac Surg. 2016;27(7):1759-64.

35. Gordon T, English AW. Strategies to promote peripheral nerve regeneration: Electrical stimulation and/or exercise. Eur J Neurosci. 2016;43(3):336-50.

36. Arbat-Plana A, Torres-Espín A, Navarro X, Udina E. Activity dependent therapies modulate the spinal changes that motoneurons suffer after a peripheral nerve injury. Exp Neurol. 2015;263:293-305.

37. Asensio-Pinilla E, Udina E, Jaramillo J, Navarro X. Electrical stimulation combined with exercise increase axonal regeneration after peripheral nerve injury. Exp Neurol. 2009;219:258-65.

38. Brandt J, Evans JT, Mildenhall T, Mulligan A, Konieczny A, Rose SJ, et al. Delaying the onset of treadmill exercise following peripheral nerve injury has different effects on axon regeneration and motoneuron synaptic plasticity. J Neurophysiol. 2015;113(7):2390-9.

39. Cobianchi S, Casals-Diaz L, Jaramillo J, Navarro X. Differential effects of activity dependent treatments on axonal regeneration and neuropathic pain after peripheral nerve injury. Exp Neurol. 2013;240:157-67.

40. Teodori RM, Betini J, Oliveira LS de, Sobral LL, Takeda SYM, Montebelo MI, et al. Swimming exercise in the acute or late phase after sciatic nerve crush accelerates nerve regeneration. Neural Plast. 2011;2011(783901):8.

\section{Acknowledgments}

To Conselho Nacional de Desenvolvimento Científico e Tecnológico (CNPq) for the research project financing.

\section{Corresponding author}

Gladson Ricardo Flor Bertolini. Rua: Universitária, 2069. Cascavel, Paraná, Brasil. Universidade Estadual do Oeste do Paraná.

Email: gladsonricardo@gmail.com

Manuscript received on July 13, 2017

Manuscript accepted on December 10, 2017

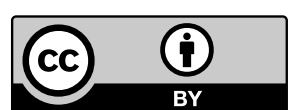

Motriz. The Journal of Physical Education. UNESP. Rio Claro, SP, Brazil - eISSN: 1980-6574 - under a license Creative Commons - Version 3.0 